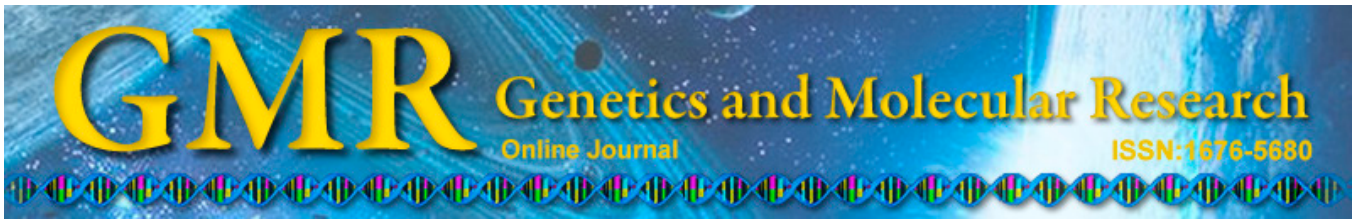

\title{
Comparative study of clinical efficacy of laparoscopy-assisted radical gastrectomy versus open radical gastrectomy for advanced gastric cancer
}

\author{
L.M. Wu, X.J. Jiang, Q.F. Lin and C.X. Jian \\ Department of Oncological Surgery, Putian Affiliated Hospital, Putian University, \\ Fujian, China \\ Corresponding author: L.M. Wu \\ E-mail: liminwucn@126.com \\ Genet. Mol. Res. 14 (2): 3459-3465 (2015) \\ Received June 25, 2014 \\ Accepted October 30, 2014 \\ Published April 15, 2015 \\ DOI http://dx.doi.org/10.4238/2015.April.15.9
}

\begin{abstract}
The purpose of this study is to compare the efficacy of laparoscopy-assisted radical gastrectomy (LARG) versus that of open radical gastrectomy (ORG). Clinical data of 355 patients who underwent radical gastrectomy (160 in the LARG group and 195 in the ORG group) were analyzed retrospectively. Efficacy indices were compared and analyzed between the two groups. The operating time of LARG was longer than that of ORG $(228.43 \pm 34.77$ versus 207.59 $\pm 28.39 \mathrm{~min})$. However, patients in the LARG group lost less blood than did those in the ORG group (169.46 \pm 82.92 versus $193.86 \pm$ $82.09 \mathrm{~mL}$ ), and more lymph nodes were removed in the LARG group (19.84 \pm 4.7 versus $18.04 \pm 4.14$ per case). The recovery of intestinal function was faster ( $3.72 \pm 1.03$ versus $4.41 \pm 1.30$ days) in the LARG group. Patients in the LARG group were administered a semi-fluid diet earlier (5.66 \pm 2.27 versus $7.09 \pm 2.33$ days) and had a shorter hospital stay $(9.44 \pm 3.06$ versus $11.07 \pm 7.91$ days $)$ than did those in the ORG group, and these differences were statistically significant $(\mathrm{P}<0.05)$. No
\end{abstract}


significant differences were found in the length of proximal and distal resection margin and the incidence of complications $(\mathrm{P}>0.05)$ between the two groups. Thus, LARG is safe, feasible, and effective for treating advanced gastric cancer.

Key words: Gastric cancer; Laparoscopy; Clinical efficacy

\section{INTRODUCTION}

Since laparoscopy-assisted radical gastrectomy (LARG) for early gastric cancer was initially reported by Japanese researchers Kitano et al. in 1994, it has been widely developed worldwide owing to its attributes of quick recovery and short hospital stay as well as mild trauma and less pain in patients. At present, the techniques of performing LARG for early gastric cancer have gradually improved and its apparent advantage - minimal invasion - has been increasingly identified. LARG has good outcomes and is defined as one of the standard therapies for stage I gastric cancer in the general rules for gastric cancer study (Japan) (Usui et al., 2003; An et al., 2010; Lee et al., 2010; Ng, 2010). However, the test for early gastric cancer is not performed as frequently in most countries as it is in Japan. In China, most patients with gastric cancer are diagnosed at the advanced stages. Therefore, the application of laparoscopy to advanced gastric cancer is necessary.

Since Goh et al. (2001) first applied laparoscopy-assisted D2 radical gastrectomy to treat advanced gastric cancer in 1997, related reports have increased year by year. However, the use of LARG for advanced gastric cancer is still in the initial stages mainly because of the rich perigastric vessels and complicated anatomical levels, which are difficult to not only expose and separate under a laparoscope but also control if massive hemorrhage occurs during surgery. Moreover, surgeons are unable to reach the gastric tissue and accurately estimate the resection edge in laparoscopy-assisted surgery as they do in open surgery. Nevertheless, D2 lymph node dissection requires the surgeons to have extensive experience in both lymph node removal and laparoscopy. Therefore, there is much dispute regarding whether LARG for advanced gastric cancer is safe and feasible, and whether the tumor can be radically removed (Allieta et al., 2009; Kato et al., 2011; Chen et al., 2012; Cui et al., 2012). Our study retrospectively analyzed and compared clinical data of patients who underwent laparoscopy-assisted or open D2 radical gastrectomy in order to explore the safety, feasibility, and clinical outcomes of LARG for advanced gastric cancer.

\section{MATERIAL AND METHODS}

\section{Patient characteristics}

Clinical data was collected from 355 advanced gastric cancer patients who underwent D2 radical gastrectomy performed by the same clinical group in the Department of Surgical Oncology, Affiliated Hospital of Fujian Putian College between January 2010 and June 2012. All patients signed informed consent forms before surgery. In all, 160 patients underwent LARG and 195 underwent open radical gastrectomy (ORG). No significant difference was found in the patient characteristics of the two groups $(\mathrm{P}>0.05)$; these data are summarized in Table 1. 


\begin{tabular}{|c|c|c|c|}
\hline General data & LARG & ORG & P value \\
\hline \multicolumn{4}{|l|}{ Gender } \\
\hline Male & 92 & 115 & \multirow[t]{2}{*}{0.779} \\
\hline Female & 68 & 80 & \\
\hline Body mass index $\left(\mathrm{kg} / \mathrm{m}^{2}\right)^{*}$ & $22.40 \pm 3.85$ & $22.20 \pm 3.75$ & 0.609 \\
\hline Age (years old)* & $58.09 \pm 10.87$ & $58.61 \pm 12.33$ & 0.675 \\
\hline \multicolumn{4}{|l|}{ Operation [case (\%)] } \\
\hline Gastrectomy & 114 & 123 & \multirow[t]{2}{*}{0.104} \\
\hline Laparoscopy-assisted distal gastrectomy & 46 & 72 & \\
\hline \multicolumn{4}{|l|}{ TNM stage [case $(\%)]$} \\
\hline IB & 35 & 36 & \multirow[t]{4}{*}{0.620} \\
\hline II & 84 & 98 & \\
\hline IIIA & 35 & 50 & \\
\hline IIIB & 6 & 11 & \\
\hline
\end{tabular}

*Values are reported as means $\pm \mathrm{SD}$.

\section{Methods of operation}

Perioperative management was conducted according to the concept of accelerated rehabilitation. During surgery, the tracheal cannula was used in patients under general and epidural anesthesia who lay in the supine position in a herringbone fashion. Perigastric lymph node dissection was performed in all patients according to the 13th Japanese edition of the D2 radical surgery standard for gastric cancer, and laparoscopic surgery was performed as described in the literature (An et al., 2010; $\mathrm{Ng}, 2010$ ). ORG was performed in the conventional way, and the D2 lymph nodes were removed according to 13th Japanese edition of general rules for gastric cancer study (Tokyo: Kanehara \& Co., Ltd., 1998). The surgeries in both the groups were performed by the same surgeons.

\section{Evaluation of indices}

The indices compared between two groups were surgical safety (rate of conversion to laparotomy, operating time, amount of bleeding, and incidence of postoperative complications), radical surgery index (number of resected lymph nodes and length of resection margin), and short-term efficacy (postoperative analgesia rate, anal ventilation time, time of administering semi-fluid diet, and postoperative hospital stay).

\section{Statistical analyses}

The experiment data were analyzed using the SPSS 14.0 software and are reported as means $\pm \mathrm{SD}$. Measurement data and enumeration data were analyzed using the Student $t$-test and chi-square test, respectively.

\section{RESULTS}

\section{Surgical safety-related indices}

No surgical death occurred in either group. Among the 160 patients in the LARG group, 157 underwent successful surgeries and 3 patients were converted to laparotomy be- 
cause of invasion of a surrounding organ by the larger tumor or owing to obesity. Compared with those in the ORG group, patients in the LARG group required longer operating time and lost less blood $(\mathrm{P}<0.05)$. In the LARG group, 1 patient with postoperative anastomotic bleeding was treated with reoperation; 2 patients with abdominal bleeding and 2 with pleural effusion were managed by conservative treatment; 5 patients with anastomotic fistula were treated by drainage and nutritional support; 3 patients with lung infection and 1 with abdominal infection were treated using strengthened anti-infection therapy; and 2 patients with ileus were cured by fasting and parenteral nutritional support. No surgical incision infection was found. In the ORG group, 2 patients had postoperative anastomotic bleeding; 5, pleural effusion; 7 , lung infections; 5 , surgical incision infection; 7, anastomotic fistula, and 5, ileus. No statistical difference was found between the incidences of postoperative complications in the two groups (Table 2).

Table 2. Comparison of surgical safety-related indices between LARG and ORG group (means $\pm \mathrm{SD}$ ).

\begin{tabular}{lccc}
\hline Indices & LARG & ORG & P value \\
\hline Total duration of surgery $(\mathrm{min})$ & $228.43 \pm 34.77$ & $207.59 \pm 28.39$ & 0.000 \\
Intraoperatve blood loss $(\mathrm{mL})$ & $169.46 \pm 82.92$ & $193.86 \pm 82.09$ & 0.006 \\
Complication [case $(\%)]$ & $16(10 \%)$ & $31(15.9 \%)$ & 0.103 \\
\hline
\end{tabular}

\section{Radical surgery index}

Surgical resection specimens of the two groups accorded with the standard, and the range of specimens and resected lymph nodes was adequate. Pathological examination of the resection margin for all specimens was negative in both groups. The lengths of the proximal and distal resection margin were $6.33 \pm 1.91$ and $5.73 \pm 1.47 \mathrm{~cm}$, respectively, in the LARG group and $6.44 \pm 2.04$ and $5.92 \pm 1.11 \mathrm{~cm}$, respectively, in the ORG group; no significant difference was found between the two groups $(\mathrm{P}>0.05)$. The number of resected lymph nodes was $19.84 \pm 4.7$ per case in the LARG group, which was higher than $18.04 \pm 4.14$ per case in the ORG group (Table 3).

Table 3. Comparison of surgery radical indices between LARG and ORG groups (means \pm SD).

\begin{tabular}{lcrr}
\hline Indices & \multicolumn{1}{c}{ LARG } & \multicolumn{1}{c}{ ORG } & P value \\
\hline Lengths of proximal resection margin (cm) & $6.33 \pm 1.91$ & $6.44 \pm 2.04$ & 0.621 \\
Lengths of distal resection margin (cm) & $5.73 \pm 1.47$ & $5.92 \pm 1.11$ & 0.149 \\
Number of removed lymph nodes/case & $19.84 \pm 4.7$ & $18.04 \pm 4.14$ & 0.000 \\
\hline
\end{tabular}

\section{Short-term efficacy indices}

Compared with patients in the ORG group, those in the LARG group experienced less pain and a shorter anal ventilation time; they were administered a semi-fluid diet earlier and had a shorter postoperative hospital stay (Table 4).

\begin{tabular}{|c|c|c|c|}
\hline Indices & LARG & ORG & $P$ value \\
\hline Postoperative analgesia requirement [case (\%)] & $77(48.1 \%)$ & $120(61.5 \%)$ & 0.011 \\
\hline Anal ventilation time (days) & $3.72 \pm 1.03$ & $4.41 \pm 1.30$ & 0.000 \\
\hline Semi-fluid diet time (days) & $5.66 \pm 2.27$ & $7.09 \pm 2.33$ & 0.000 \\
\hline Postoperative stay in hospital (days) & $9.44 \pm 3.06$ & $11.07 \pm 7.91$ & 0.014 \\
\hline
\end{tabular}




\section{DISCUSSION}

In recent years, LARG has been increasingly used as one of the standard treatments for stage I gastric cancer in Japan and Korea among other countries. However, in most countries, majority of patients with gastric cancer are diagnosed at an advanced stage. Thus, several surgeons are trying to apply laparoscopy to the treatment of advanced gastric cancer. In this study, we gradually developed the laparoscopic technique for advanced gastric cancer based on the abundant experience in laparoscopy for early gastric cancer, and compared it with ORG to explore surgical safety and the radical nature of laparoscopyic operations for advanced gastric cancer.

Complete resection of lesions and adequate resection margin are the principles that radical gastrectomy must comply with (Ahmad et al., 2007). In China, the recommended adequate resection margin is more than $5 \mathrm{~cm}$. In our study, the lengths of proximal and distal resection margin were $6.33 \pm 1.91 \mathrm{~cm}$ and $5.73 \pm 1.47 \mathrm{~cm}$, respectively, in the LARG group, showing no significant difference compared with those of the ORG group $(\mathrm{P}>0.05)$, which was consistent with foreign literature. Pugliese et al. (2007) reported that the average length of distal resection margin was $6.8 \mathrm{~cm}$ in 19 patients with advanced gastric cancer who underwent laparoscopy-assisted D2 radical gastrectomy, and they showed no residual cancer at the postoperative stump. Chun et al. (2012) demonstrated that the mean length of the distal resection margin was $5.0 \mathrm{~cm}$ in 52 cases of pT2 gastric cancer treated with LARG. In addition, approximately $1.0 \mathrm{~cm}$ of distal residue was present in the anvil block of the anastomat used in surgery, which indicated that laparoscopy ensured the safety of resection margin as ORG did.

The number of dissected lymph nodes has been considered as an important index in the evaluation of radical surgery for gastric cancer (Shiraishi et al., 2006; Seevaratnam et al., 2012). Miura et al. (2004) categorized and compared the number of resected lymph nodes between the LARG and ORG groups, and discovered that the number of resected lymph nodes in the LARG group, especially in the 4th, 6th, 9th, and 11th groups were markedly lesser than those in the ORG group due to difficulties in operation. However, Huscher et al. (2005) showed a similar number of resected lymph nodes in both groups by using the same analysis. Moreover, Huang et al. (2011) demonstrated that the average number of resected lymph nodes (29.1 \pm 10.4 per case) in the LARG group was similar to that of ORG group, except that the number of 7 th and 8 th lymph nodes was distinctly greater in the LARG group. In this research, no significant difference was found in the number of resected lymph nodes between the two groups $(\mathrm{P}>0.05)$. We identified skilled laparoscopic technique and good sense of anatomical hierarchy as the key points for lymph node resection. Therefore, the number of resected lymph nodes should be similar as long as the operation was performed according to standard surgical steps for radical surgery for gastric cancer. LARG can result in an outcome similar to that of ORG.

Operating time, intraoperative blood loss, incidence of complication and mortality are significant indices for evaluating surgical safety (Ahmad et al., 2007). In our study, 3 patients $(1.88 \%, 3 / 160)$ were converted to laparotomy because of invasion of a surrounding organ by the larger tumor or owing to obesity, and no death occurred. Operating time and intraoperative blood loss in LARG for advanced gastric cancer was closely correlated with the laparoscopic experience of surgeons. In this research, the LARG group had a longer operating time but lost less blood compared with the ORG group, and this helped the patients undergoing LARG with quick postoperative recovery. Apart from the pneumoperitoneum-related complication of LARG, other complications are similar to those of ORG. A retrospective study of LARG 
in 1485 cases from 10 centers in Korea revealed that the incidence of complications and mortality were 14 and $0.6 \%$, respectively, for LARG (Kim et al., 2008) and 14.4 and $0-1.2 \%$, respectively, for ORG (Moriwaki et al., 2001). According to the foreign literature, the incidence of complications related to LARG was lower than that of ORG. Although we did not find any such difference between the two groups in our study $(\mathrm{P}>0.05)$, the incidence of complications was lesser in LARG (10 to $15.9 \%$ ), indicating that LARG was safe for patients with advance gastric cancer. We believe that the incidence of complications in LARG mainly correlated with the laparoscopic operation technique used by the surgeons and their proficiency in performing laparoscopy. In order to reduce the surgical complications and the rate of conversion to laparotomy, doctors should check for surgical indications and perform a careful preoperative evaluation. Peritoneoscopy should be performed to assess the cases in which it is difficult to determine the stage before surgery. Conversion to laparotomy should be conducted in patients who are not suitable for LARG, and surgeons must not violate the surgical principle for minimal invasion.

The main criterion for evaluating whether a new surgical technique is superior to conventional methods is the clinical benefit in patients. Our research demonstrated that compared with those in the ORG group, patients in the LARG group experienced lesser pain and shorter anal ventilation time; they were administered a semi-fluid diet earlier and had a shorter postoperative hospital stay. At present, our study as well as previous reports (Miura et al., 2004; Huscher et al., 2005; Huang et al., 2011) demonstrated several advantages of LARG for advanced gastric cancer, including mild trauma, quick postoperative recovery and short hospital stay, indicating that the patients who underwent LARG had a better early life quality than did those who underwent ORG. As for the long-term life quality of patients who underwent LARG, Yasuda et al. (2007) discovered that the total score of 22 indicators, such as daily eating frequency, food intake at each meal and appetite, in the LARG group was similar to that of the ORG group; however, the incidence of postoperative ileus in the LARG group (1\%) was distinctly lower than that in the ORG group (13\%).

In conclusion, LARG is a safe, feasible, and effective method for the treatment of advanced gastric cancer. LARG is characterized by less intraoperative blood loss, high number of resected lymph nodes, and quick postoperative recovery, as well as a radical efficacy similar to that of ORG.

\section{REFERENCES}

Ahmad Z, Idrees R, Azad NS, Ahmed R, et al. (2007). Gastric carcinoma: typing, staging, lymph node and resection margin status on gastrectomy specimens. J. Coll. Physicians Surg. Pak. 17:539-542.

Allieta R, Nardi M Jr, Brachet-Contul R, Millo P, et al. (2009). Laparoscopic gastrectomy for treatment of advanced gastric cancer: preliminary experience on 38 cases. Minerva Chir. 64: 445-456.

An JY, Heo GU, Cheong JH, Hyung WJ, et al. (2010). Assessment of open versus laparoscopy- assisted gastrectomy in lymph node-positive early gastric cancer: a retrospective cohort analysis. J. Surg. Oncol. 102: 77-81.

Chen QY, Huang CM, Lin JX, Zheng CH, et al. (2012). Laparoscopy-assisted versus open D2 radical gastrectomy for advanced gastric cancer without serosal invasion: a case control study. World J. Surg. Oncol. 10: 248.

Chun HT, Kim KH, Kim MC and Jung GJ (2012). Comparative study of laparoscopy- assisted versus open subtotal gastrectomy for pT2 gastric cancer. Yonsei Med. J. 53: 952-959.

Cui M, Xing JD, Yang W, Ma YY, et al. (2012). D2 dissection in laparoscopic and open gastrectomy for gastric cancer. World J. Gastroenterol. 18: 833-839.

Goh PM, Khan AZ, So JB, Lomanto D, et al. (2001). Early experience with laparoscopic radical gastrectomy for advanced gastric cancer. Surg. Laparosc. Endosc. Percutan. Tech. 11: 83-87.

Huang CM, Lin JX, Zheng CH, Li P, et al. (2011). Effect of laparoscopy assisted vs open radical gastrectomy on lymph node dissection in patients with gastric cancer. Chin. J. Surg. 49: 200-203. 
Huscher C, Mingoli A, Sgarzini G, Sansonetti A, et al. (2005). Value of extended lymphadenectomy in laparoscopic subtotal gastrectomy for advanced gastric cancer. J. Am. Coll. Surg. 200: 314.

Japanese Gastric Cancer Association (1998). Japanese classification of gastric carcinoma, 13th edn (in Japanese). Kanehara \& Co., Ltd., Tokyo, 101-112.

Kato K, Kiyokawa T, Mori K, Fukuda T, et al. (2011). Current state of treatment for advanced gastric cancer. Gan To Kagaku Ryoho. 38: 184-186.

Kim MC, Kim W, Kim HH, Ryu SW, et al. (2008). Risk factors associated with complication following laparoscopyassisted gastrectomy for gastric cancer: a large-scale Korean multicenter study. Ann. Surg. Oncol. 15: 2692- 2700.

Kitano S, Iso Y, Moriyama M and Sugimachi K (1994). Laparoscopy-assisted Billroth I gastrectomy. Surg. Laparosc. Endosc. 4: 146-148.

Lee SW, Nomura E, Bouras G, Tokuhara T, et al. (2010). Long-term oncologic outcomes from laparoscopic gastrectomy for gastric cancer: a single-center experience of 601 consecutive resections. J. Am. Coll. Surg. 211: 33-40.

Miura S, Kodera Y, Fujiwara M, Ito S, et al. (2004). Laparoscopy-assisted distal gastrectomy with systemic lymph node dissection: A critical reappraisal from the viewpoint of lymph node retrieval. J. Am. Coll. Surg. 198: 933-938.

Moriwaki Y, Kobayashi S, Kunisaki C, Harada H, et al. (2001). Is D2 lymphadenectomy in gastrectomy safe with regard to the skill of the operator? Dig Surg. 18: 111-117.

Ng KK (2010). Laparoscopy-assisted gastrectomy for early gastric cancer is safe and effective in elderly patients. J. Surg. Res. 158: 30-32.

Pugliese R, Maggioni D, Sansonna F, Scandroglio I, et al. (2007). Total and subtotal laparoscopic gastrectomy for adenocarcinoma. Surg. Endosc. 21: 21-27.

Seevaratnam R, Bocicariu A, Cardoso R, Mahar A, et al. (2012). A meta-analysis of D1 versus D2 lymph node dissection. Gastric Cancer 15: 60-69.

Shiraishi N, Yasuda K and Kitano S (2006). Laparoscopic gastrectomy with lymph node dissection for gastric cancer. Gastric. Cancer 9: 167-176.

Usui S, Inoue H, Yoshida T, Fukami N, et al. (2003). Hand-assisted laparoscopic total gastrectomy for early gastric cancer. Surg. Laparosc. Endosc. Percutan. Tech. 13: 304- 307.

Yasuda K, Shiraishi N, Etoh T, Shiromizu A, et al. (2007). Long-term quality of life after laparoscopy-assisted distal gastrectomy for gastric cancer. Surg. Endosc. 21:2150-2153. 